Raman Effect and Electrolytic Dissociation.

A STUDY of the Raman effect in nitric acid at various concentrations has revealed two interesting features which have a bearing on the phenomenon of electrolytic dissociation. In concentrated nitric acid (about 65 per cent acid), nine Raman lines have been found, three of which correspond to water. Of the others, three lines showing wave number differences of 958,1312 , and $3319 \mathrm{~cm} .^{-1}$ seem to belong to the $\mathrm{HNO}_{3}$ molecule. The other three, which are also found in nitrates, with wave-number differences of 630, 689, and $1050 \mathrm{~cm}^{-1}$ belong to the $\mathrm{NO}_{3}{ }^{-}$ion. Photographs taken indicate that the Raman lines belonging to the $\mathrm{HNO}_{3}$ molecule gradually disappear with increasing dilution. On the other hand, the lines belonging to the $\mathrm{NO}_{3}^{-}$ion increase in brightness up to a certain dilution and then diminish with still further dilution. The dilution corresponding to the maximum brightness of the $\mathrm{NO}_{3}{ }^{-}$lines is not far from that at which the $\mathrm{HNO}_{3}$ lines just disappear. This is clear evidence of the dissociation of the $\mathrm{HNO}_{3}$ molecule.

Water, which gives three diffuse bands at 3208 , 3419 , and $3582 \mathrm{~cm}^{-1}$ wave number differences, shows a peculiar behaviour. Not only do the three bands become sharper with increasing concentration of the acid, but the band corresponding to $3208 \mathrm{~cm} .^{-1}$ gradually diminishes in intensity until it becomes very faint in the concentrated acid. The band $3582 \mathrm{~cm}^{-1}$, which is the weakest of the three in pure water, gains rapidly in intensity and becomes in the concentrated acid the strongest of the three bands.

It is expected that intensity measurements, which are in progress, will throw light on the current theories of electrolytic dissociation.

Wheatstone Laboratory, King's College,

London, Oct. 9.

\section{RAMAKRISHNA RAO.}

\section{Molecular Spectra and Molecular Structure.}

Those who contributed to the recent Faraday Discussion on molecular spectra and molecular structure have now sent their written versions to the secretary for publication. Instead of the report we gave of Prof. Barker's explanation of the double $Q$ branch of ammonia at $10.3 \mu$ and $10.7 \mu$ (NATURE, Oct. 12, p. 587), we should like to substitute Prof. Barker's own written version :

"The ammonia molecule probably has the form of a triangular pyramid of small altitude. Two of the four fundamental modes of vibration involve an oscillation of the $\mathrm{N}$ atom along the axis of symmetry, i.e. normal to the plane of the three $\mathrm{H}$ atoms. The band at $10.5 \mu$ is associated with a symmetrical motion of the $H$ atoms such that the distance between them increases when the $\mathbf{N}$ atom approaches the plane in which they lie. The equilibrium position of the $\mathrm{N}$ atom may be upon either side of this plane; hence the potential energy function exhibits two minima separated by a relatively low maximum. Two eigenfunctions must therefore be associated with each vibration state, one being symmetrical and the other anti-symmetrical. Because the two equilibrium positions are not far apart these two functions involve slightly different amounts of energy, and the vibration levels are all double. Finally, since transitions are always between states of opposite symmetry character, e.g. $1_{a} \rightarrow 2_{s}$ or $1_{s} \rightarrow 2_{a}$, two absorption bands appear with slightly different frequencies."

W. E. Garner.

J. E. LenNARD-Jones.

The University, Bristol.

No. 3133, VoL. 124]

\section{New Cellulose Methylene Ether.}

SYMmetrical dichlorodimethyl sulphate isolated by Fuchs and Katscher (Ber., 60, 2288; 1927) has been found to react with soda cellulose (using Haworth's technique) giving monomethylene cellulose of maximum $\mathrm{OCH}_{2}$ content 17.2 per cent and corresponding with the formula $\mathrm{C}_{6} \mathrm{H}_{7} \mathrm{O}_{2}(\mathrm{OH})\left(\mathrm{O}_{2} \mathrm{CH}_{2}\right)$.

Other methylene inorganic and organic esters give somewhat similar results. This reaction is evidently exactly analogous to that between soda cellulose and dimethyl sulphate, except that only two of the three hydroxyl groups of the $\mathrm{C}_{6} \mathrm{H}_{10} \mathrm{O}_{5}$ unit take part in it.

Hitherto, all work on the reaction between cellulose or starch and formaldehyde has given either the socalled ' absorption compounds ', or with acid catalysts only partially methyleneated products. Two recent papers on this subject deal with these aspects of the reactions (Blanksma, Rec. Trav. Chim., 361 ; 1929, and Meunier and Guyot, Comptes rendus, 188, 506; 1929). It is hoped to be able to establish the structure of monomethylene cellulose now described for the first time, and particularly to apply the reaction to many of the sugars and starches.

Monochlorodimethyl sulphate reacts with soda cellulose giving a mixed methyl methylene cellulose ether of limited interest only.

\section{Egerton Road, Fallowfield,} Manchester, Oct. 18

\section{Influence of Particle Size on Diamagnetism.}

IN a recent note to Nature (July 13, p. 53), Sir C. V. Raman referred to some experiments made with me which showed that the diamagnetic susceptibility of graphite falls off steadily with increasingly fine subdivision of the substance. A single crystal of graphite gave a specific susceptibility $\left(x-10^{6}\right)$ equal to $18 \cdot 1$ parallel to the hexagonal axis, and $2 \cdot 7$ perpendicular to it, the average over all directions being $7 \cdot 8$. With powdered graphite this number diminishes to $5 \cdot 1$, while for finely divided colloidal graphite it falls so low as $2 \cdot 2$.

Further experiments by me have disclosed a similar effect with metallic antimony, though not of such a striking character. Massive antimony has a specific susceptibility $\left(x-10^{6}\right)$ equal to $0 \cdot 78$, which diminishes to 0.71 with colloidal antimony when the average particle size is $6 \mu$ and further drops to 0.54 with particles $150 \mu \mu$ in size. It is known that the diamagnetic susceptibility of antimony falls off with rise of temperature, or on fusion, and that it is considerably less for antimony in chemical combination than for the free metal. It appears that mechanical subdivision or 'colloidalisation' influences the diamagnetism in the same way.

$$
\text { V. I. VAIDYANATHaN. }
$$

Annamalai University,

Chidambaram, South India, Oct. 1.

\section{Periodic Precipitations and Diffusion.}

IN a recent communication (Proc. Roy. Irish Acad., 38 B, p. 445) we classed Prof. Wolfgang Ostwald's theory of periodic precipitations amongst those in which precipitation should occur at or near the " head of the diffusion column ". In reality, as Prof. Ostwald has kindly pointed out to us in a private communication, according to his theory the "critical mixture ratio", which determines the formation of a precipitate, should always lag behind the head of the diffusion column. This objection to Ostwald's theory is therefore not valid.

Hugh RYAN.

University College, Dublin.
R. J. DOYLE. 